\title{
The merit of using silicon for the development of hearing aid microphones and intraocular pressure sensors*
}

\author{
P. Bergveld \\ MESA Research Institute, Unversity of Twente, Box 217, 7500 AE Enschede (Netherlands)
}

\begin{abstract}
An important design rule for a hearing aid is the requirement of a large signal to noise ratio, which is mainly determined by that of the microphone and its preamplifier It will be shown that in order to increase the signal to noise ratio it is favourable to integrate the preamplifier with the microphone, which implies that the microphone should be made of slicon, preferably with a single wafer technology For the development of a tonometer for the measurement of intraocular pressure, the application of a silicon force sensor rationalizes that also the flattening of the eye globe is measured with a silicon applanation sensor, instead of by optical means which is the present practice $A$ sensor construction has been developed, which combines a force, pressure and applanation sensor, all made in silicon
\end{abstract}

\section{Introduction}

Besides the various ISFET-related biosensor projects, focused on the dynamic stimulus-response measurement, the biosensor group of the MESA Research Institute is also active in the area of physical sensors for biomedical applications In this paper attention will be paid to the advantage of using silicon micromachıning and integrated electronic circuitry for the development of a microphone for heanng ands and a tonometer pen with electronic read-out for determining open-angle glaucoma

\section{The hearing and components}

Present hearing aids are produced by companies which buy the essential components the receiver (telephone), the amplufier and the microphone from spec1alızed companies The recerver is always from the electromagnetic type, due to the required output power The most modern receiver contains a built-in power amplifier The hearing-and amplifier serves the microphonc signal amplification and filtering, often with remote control The microphone is always of the electret type, because of its very high sensitivity Due to its capacitive nature, the microphone capsule needs to contam a bult-in impedance transformer Figure 1 shows a block diagram of a complete hearing ald in which the essential parts as just described can be distınguished

\footnotetext{
*Invited paper
}

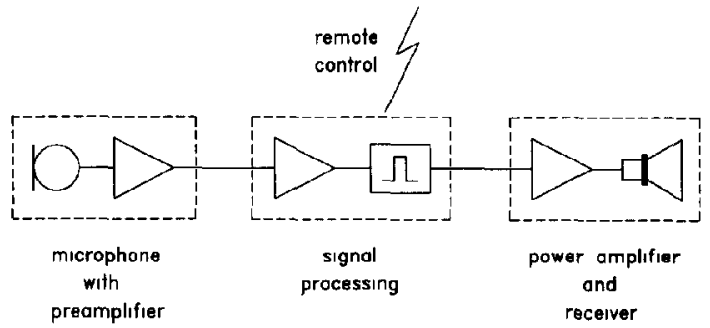

Fig 1 Block diagram of a modern hearing atd

The electro-acoustical quality of a hearing aid is mainly determined by its power consumption, its frequency charactenstic, its distortion and its signal-tonoise ratio Improvement of these parameters is one of the present desires of hard of heanng patients

The distortion is mainly caused by the electromagnetic receiver, of which the required power is obtained by making use of resonance peaks in the transfer characteristics Up to now it has not appeared to be possible to obtain the required power by other, less distorting transduction principles

To minimize the power consumption, the power amplifier is of the class $D$ type, whilst low voltage CMOS circuits are used for the signal amplification and processing

As with all measuring systems noise reduction can most effectively be realized by increasing the $S / N$ ratio of the first stage, in this case the microphone and its preamplifier Therefore electret microphones are used, of which at present the development is focused on 
increasing the sensitivity, maintaining at the same time the required frequency range of $100 \mathrm{~Hz}$ to $7 \mathrm{kHz}$ at a minimum noise level As will be discussed in the next section, integration of the mucrophone and the preamplifier favours the $S / N$ ratio, which fact underlines the usefulness of developing condenser microphones in silicon, using micromachining techmiques

The noise in microphones with a built-in preamplifier

The conventional electret condenser microphones consist of a stainless steel backplate with acoustic holes to which a teflon electret is attached and which is separated by an air gap from a Mylar membrane The microphone is mounted in a metal case, which also contains a hybrid JFET source follower, including a gate bias resistor van der Donk et al calculated the equivalent input noise density $N_{1}\left(\mathrm{~V} / \mathrm{Hz}^{1 / 2}\right)$ of such a system $[1,2]$ For low frequencies it could be calculated that

$N_{1}^{2}=\left(\frac{C_{\mathrm{m}}+C_{\mathrm{par}}}{C_{\mathrm{m}}}\right)^{2}\left(\frac{K}{f}+\frac{8 k T}{3 g_{\mathrm{m}}}\right)+\frac{4 k T\left(1 / R_{\mathrm{b}}+1 / R_{\mathrm{g}}\right)}{(2 \pi)^{2} C_{\mathrm{m}} 2}$

where $C_{\mathrm{m}}$ is the microphone capacitance, $C_{\mathrm{par}}$ is the sum of all parasitic capacitances, $K$ is the flicker noise coefficient, $f$ is the frequency, $g_{\mathrm{m}}$ is the transistor mutual conductance, $R_{\mathrm{b}}$ is the bias resistor, $R_{\mathrm{g}}$ is the (frequency dependent) resistor representing the encapsulation resistance of the hybrid preamplifier and $k T$ has the usual meaning As can be seen from eqn (1) the bias resistor $R_{\mathrm{b}}$ and the parallel, parasitic encapsulation resistor should be as high as possible, as well as the microphone capacitance $C_{m}$, whilst parasitic (loading) capacitances should be as low as possible in order to minimize the noise performance of the system Experments have shown that in practice the value of $R_{b}$ need not be chosen higher than $7 \mathrm{G} \Omega$, because at higher values the frequency dependent encapsulation resistance is dominating Further reduction of the noise can only be achieved by getting nd of this encapsulation resistance and thus by integration of the preamplifier and the microphone, meanwhile also decreasing the parasitic capacitances This is the main reason that in the future slicon should be used as the substrate material for condenser microphones The exploration of vanous micromachining techniques, like anisotropic etching and sacrificial layer technology as developed in the last decade, will be of great value in this respect The possibilities of both techniques will be illustrated in the next sections

\section{Anisotropically etched silicon backplates}

In order not to make too large steps in the development of silicon electret microphones, we decided in 1982 to focus in one of the $\mathrm{Ph} \mathrm{D}$ projects (Sprenkels) in the first place on wafer scale production of slicon backplates [3, 4] The advantage with respect to the conventional stanless steel backplates is the flexibility of the design Just by changing the mask design one can easily change the dimensions and the number of acoustic holes, as well as the thickness of the air gap, which is in this case a slicon etched cavity The desired frequency response in relation to sensitivity can in this way be fixed beforehand by the mask design Moreover, many identical backplates can be manufactured in this way

In the first design we made use of the conventional heat sealing of a tefion foil to the backplate, identical to the process in use for the stainless steel backplates Also the Mylar foil as used for conventional microphones was used as the microphone diaphragm, glued onto the backplate by a polymer spray via glueholes, etched especially for this purpose into the backplates The resulting microphone is shown in Fig 2

Parallel to the development of silicon backplates, an unvestigation was carned out concerning the possibilities of replacing the teflon electret by a silicon dioxide electret, because the teflon sealing process is not very IC compatıble and silicon dioxıde can so easily be produced on silicon backplates It appeared that a silicon dioxide layer with a thickness of the order of $1 \mu \mathrm{m}$ can operate as an excellent electret, provided that a chemical treatment with a hydrophobic agent, such as HMDS, is carned out to prevent charge compensation through water induced surface conductivity [5] At the end of the Ph D project of Sprenkels it was proven that silicon microphones with a silicon dioxide electret, charged up to $200 \mathrm{~V}$, an airgap of 30 $\mu \mathrm{m}$ and a Mylar membrane could be produced in large quantities, having an open circuit sensitivity (without preamplifier attenuation) of $25 \mathrm{mV} / \mathrm{Pa}$ and a flat frequency charactenstıc larger than $15 \mathrm{kHz}$ Both parameters fulfill the requirements of hearing alds

Integration of this type of slicon microphone with a CMOS amplifier is however difficult, due to the

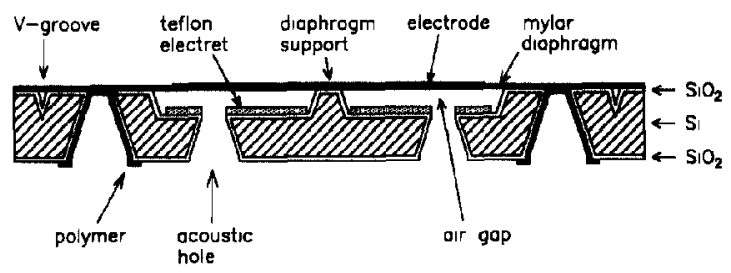

Fig 2 Silıcon microphone after Sprenkels [3] 
incompatibility of the corona charging of the electret with electronic circuitry Therefore it was decided to develop a microphone with a thinner air gap, making a lower bias voltage possible, which can be derived from the $12 \mathrm{~V}$ hearing ald battery by means of an integrated voltage multiplier In addition to this new microphone design, the Mylar membrane should be replaced by a CMOS compatible material and process Exploitation of the sacrificial layer technique appeared to be the solution, as shown in the $\mathrm{Ph} \mathrm{D}$ project of Scheeper [6] and summarized in the next section

\section{Microphone design based on the sacrificial layer technique}

Instead of etching the air gap as a cavity in the slicon backplate, the air gap can also be realized by depositing and shapıng an etchable layer onto the silicon surface and covering this with the diaphragm material. The sacrificial layer can be etched away through access holes, which can serve later on as the acoustical holes A layer of aluminium with silicon nitnde appears to be a suitable combination The resulting microphone is shown in Fig 3

One of the difficulties experienced with the rather simple technique is the sticking of the membrane to the backplate after the etch process [7] Using a freeze drying procedure this problem could be solved

A more senous drawback of the design is the intrinsic large ardamping, which limits the high frequency behaviour Due to the shape of the anisotropically etched holes in the backplate, with the typical angle of $547^{\circ}$ for $\langle 100\rangle$ silicon, the number of holes is rather limited, resulting in a relatively large lateral air streaming resistance Therefore an alternative design has been developed, as shown in Fig $4[8,9]$

Instead of using silicon, a layer of silicon nitride is used as the backplate, which opens up the possibility of using a very large number of acoustic holes The rigid silicon substrate now serves as the membrane support Therefore the silicon has first to be provided with a layer of LPCVD silicon nitnde, before the aluminium sacrificial layer is deposited Over this layer a $1 \mu \mathrm{m}$ PECVD silicon nitnde layer is deposited, as

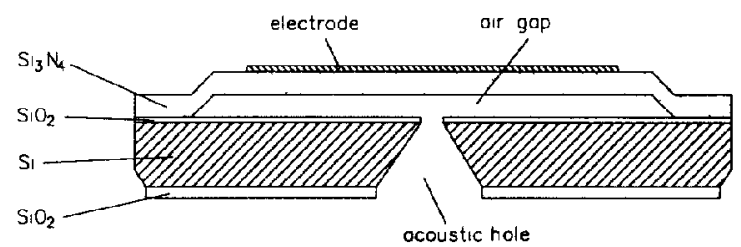

Fig 3 Silicon microphone design based on the sacrificial layer techmque

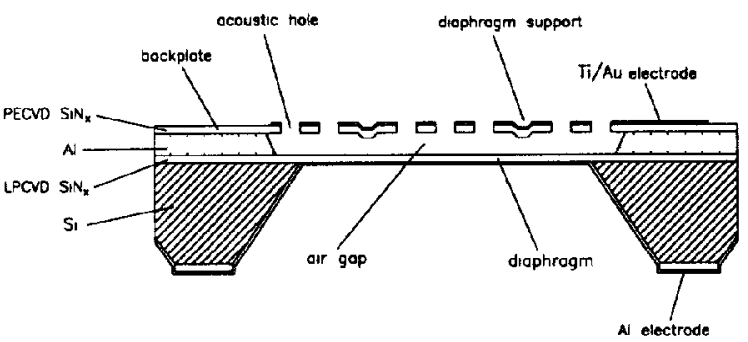

Fig 4 Microphone design with silicon nitride backplate

well as a layer of gold $(30 \mathrm{~nm}$, over a $30 \mathrm{~nm}$ titanium adhesion layer) The alumınum sacrificial layer is etched through a large number of access holes in the gold/ titanum/silicon nitride layer, which later on serve as the acoustic holes

The actual microphone diaphragm is the first layer of LPCVD silicon nitride, etched free by an anisotropic etch from the backside, before the aluminium is deposited By adding small irregularities in the backplate, the sticking of the two layers of silicon nitride after etching the sacrificial layer could be largely prevented

A typical microphone with a $2 \times 2 \mathrm{~mm}$ diaphragm and backplate of $1 \mu \mathrm{m}$ silicon nitnde, having an airgap of $31 \mu \mathrm{m}$, an external bias voltage of $5 \mathrm{~V}$ and 242 acoustic holes, shows a sensitivity of $78 \mathrm{mV} / \mathrm{Pa}$ over a frequency range up to $14 \mathrm{kHz}$ It shows that the frequency response now satisfies the requirements for hearing aids, but also that the sensitivity is not yet sufficient

Note that the backplate in this new microphone design consists of a highly perforated metallized layer of slicon nitride, instead of the shaped silicon substrate as in the original design

The great advantage of this new fabrication process is that it is a planar, relatively low temperature process, very suitable for carrying out after a CMOS process In the $\mathrm{Ph} \mathrm{D}$ project of van der Donk it is proven in the meantime that a PMOS source follower with diode bias element, as well as a CMOS Dickson voltage converter, are suitable electronic circuits to be integrated [10]

\section{Future developments}

As described in the previous sections, the integration of electronic functions with a surface micromachined microphone seems possible because of the compatibility of the processes However the microphone should have a higher sensitivity Future developments have therefore to be focused on increasing the sensitıvity In order to do so, the microphone operation has been modelled to find the optimization parameters of which the results are summanzed below [11] 
The sensitivity $S$ of a condenser microphone can be described as

$S=-S_{\mathrm{e}} S_{\mathrm{m}} H$

where $S_{\mathrm{e}}$ is the electrical sensitivity, $S_{\mathrm{m}}$ the mechanical sensitivity of the diaphragm and $H$ the electrical transfer characteristic of the source follower, including the attenuation effect of the capacitive loading In the first instance eqn (2) suggests that one should strive after $H=1$ (large $C_{\mathrm{m}}$ and small parasitıc loading capacitance), with a large value of $S_{\mathrm{c}}$ as well as $S_{\mathrm{m}}$ It appears, however, that $S_{c}$ and $S_{\mathrm{m}}$ cannot be maximized independently

The electrical sensitivity is given by

$S_{\mathrm{e}}=E_{\mathrm{a}}=\frac{V_{\mathrm{b}}}{S_{\mathrm{a}}}$

where $E_{\mathrm{a}}$ is the electrical field in the air gap, $V_{\mathrm{b}}$ the microphone bias voltage and $s_{\mathrm{a}}$ the air gap thickness Increasing the electncal sensitivity by using a higher bias voltage 15 , however, limited by the possible collapse of the diaphragm to the backplate, due to the static attraction forces

The mechanical sensitivity is given by

$S_{\mathrm{m}}=\frac{R^{2}}{8 \sigma_{\mathrm{d}} h_{\mathrm{d}}}$

where $R$ is the radius of a circular diaphragm, $\sigma_{\mathrm{d}}$ the diaphragm stress and $h_{\mathrm{d}}$ the diaphragm thickness Equation (4) shows that the mechanical sensitivity $S_{\mathrm{m}}$ of a diaphragm with a certain radius and initial stress can be increased by decreasing its thickness For a condenser microphone this is however a limited possibility to increase the value of $S$, because increasing $S_{\mathrm{m}}$ will simultaneously decrease the collapse voltage, according to eqn (5), forcing the use of a lower value for $E_{\mathrm{a}}$ and thus $S_{\mathrm{e}}$

$V_{\text {collapse }}=\left(\frac{1}{S_{\mathrm{m}}} \frac{8 s_{\mathrm{a}} 2}{27 \epsilon_{0}}\right)^{1 / 2}$

The considerations mentioned above show that the electrical sensitivity and the mechanical sensitivity cannot be increased independent of each other Nevertheless, combination of eqns (2), (3), (4) and (5) shows that, by adapting the bias voltage to a fixed fraction of the collapse voltage (say $60 \%$ ), the sensitivity $S$ of a condenser microphone can be increased by increasing the mechanical sensitivity of the diaphragm $S$ will increase proportional to the square root of the mechanical sensitivity

Increasing the mechanical sensitivity of the diaphragm by decreasing its thickness, independent of the difficult to control diaphragm stress, may however be troublesome A solution to this problem may be the introduction of corrugations in the diaphragm Preliminary results have shown that this is a realistic approach [12] The mechanical sensitivity of a corrugated diaphragm was measured to be 25 times the sensitivity of the related flat diaphragm A further advantage of the application of corrugations in a diaphragm is that the influence of temperature and packaging stress will be greatly reduced

\section{Electronic tonometers}

Ophthalmologists measure the intraocular pressure $(I O P)$ of their patients in order to diagnose the eye disease open-angle glaucoma Normal pressures are in the range $10-15 \mathrm{~mm} \mathrm{Hg}$, whilst a pressure of over 21 $\mathrm{mm} \mathrm{Hg}$ may indicate the above mentioned disease

Based on the simple law that $P=F / A$, the internal pressure $P$ in a sphere can be calculated by measuring the necessary force $F$ that can flatten a certain area $A$ of the sphere Application of this law to IOP measurements has shown that two correction terms have to be introduced, one duc to the cornea stiffness and the other resulting from shear forces due to the cornea tear film It appeared that both correction terms cancel each other if the flattened area has a diameter of 306 mm Based on this knowledge Goldmann developed in 1957 a tonometer, an instrument which is at present still in use

The Goldmann tonometer consists of a force transducer to measure the force by which a glass plate is pressed against the anaesthetized comea Because the anaesthetizing fiurd is made fluorescent, the edges of the applanation can be observed by means of a slitlamp microscope With this optical system the diameter of the applanation can be measured and adjusted to 306 $\mathrm{mm}$ From the corresponding force the IOP can be calculated

Another approach to measure the $I O P$ was developed by Mackay and Marg in 1959 The Mackay-Marg tonometer uses a footplate of $5 \mathrm{~mm}$ diameter, containing a small pressure sensor in the centre Placed on the anaesthetized eye globe, a pressure versus time curve can be recorded which shows a typical dip at the value of the IOP Mackay and Marg proved that their method is independent of the cornea stuffness and the surface tension of the tear film on the cornea At a sufficient applanation radius the corresponding forces act only on the footplate and not on the central pressure sensor However, the measurement is strongly dependent on the distance between the edge of the applanation and the pressure sensor If the smallest distance between the pressure sensitive area of the sensor and the nearest edge of the applanation exceeds $1 \mathrm{~mm}$, the measurement is false, but the problem is that this critical distance 
is not monitored In practice the only criterion is that If no dip in the pressure-time curve can be detected, the measurement has to be repeated, without guarantee that the new measurement will be successful

From the description of the two most often used tonometers, the Goldmann and the Mackay-Marg tonometer, it is obvious that the development of a sensor for the monitoring of the applanation will be of great importance Moreover if this sensor can also provide information about the distance between the edge of the applanation and the centre of the footplate, this would be welcome to increase the reliablity of the Mackay-Marg system

Because at present force as well as pressure sensors are mostly made in silicon, it would be useful to develop an applanation sensor making use of silicon and the silicon technologies This would greatly enhance the possibility of integrating the force or pressure sensor with the applanation sensor Therefore in the $\mathrm{Ph} D$ project of den Besten a silicon applanation sensor has been developed, to be applied in conjunction with a force sensor for a Goldmann tonometer and in conjunction with a pressure sensor for a Mackay-Marg tonometer [13] It was decided to apply as a force sensor the intracranial pressure sensor chip as originally developed by Philips, because of its excellent specifications and its suitable dimensions of $42 \times 42 \mathrm{~mm}$ Using this sensor attached to the boss of its diaphragm it will operate as a force sensor

\section{The applanation sensor}

The applanation sensor, as mentioned in the previous section, consists of a slicon substrate of $63 \times 63 \mathrm{~mm}$, containing four meander shaped diffused resistor arrays, having 76 resistors of $13 \mathrm{k} \Omega$ each and with meander contacts at the resistor nodes $[14,15]$ The arrays are shaped around a plunger, which is located in the centre of the sensor as shown in Fig 5(a) The meander contacts are extended with a gold line pattern on the sensor surface as shown in Fig 5(b)

The sensor is covered by a $6 \mu \mathrm{m}$ thick Mylar foll, which is coated with a thin gold layer at the side which faces the sensor surface The foil is separated from the sensor by a polyumide spacer as shown in Fig 6 In the centre of the sensor a small plunger is realized, suspended in a silicon mitride membrane, realized by anisotropic etching of the slicon substrate from the backside This plunger provides the coupling with the attached force sensor, makıng the central pressure measurement possible as necessary for the Mackay-Marg method

When the applanation sensor is pressed against the eye globe, the Mylar foll with its gold film contacts a

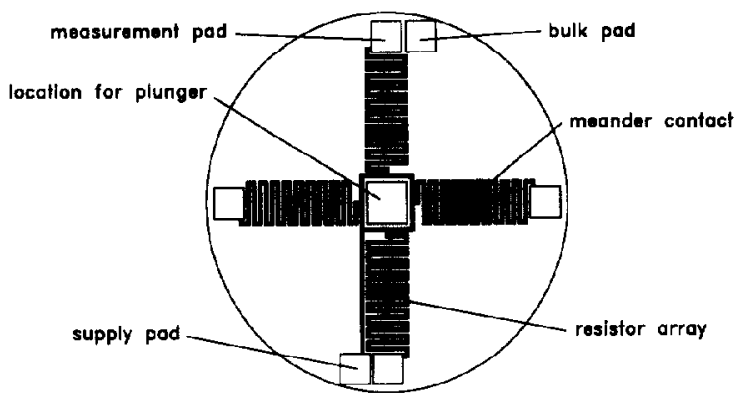

(a)

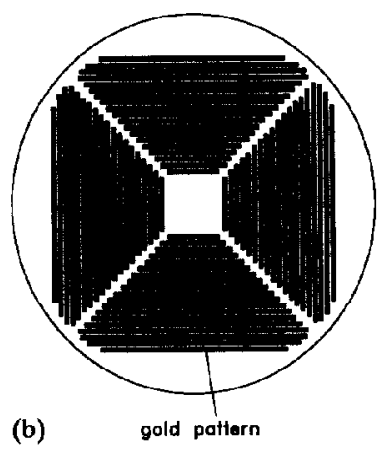

Fig 5 (a) Resistor configuration of the applanation sensor (b) Gold pattern on the surface of the applanation sensor

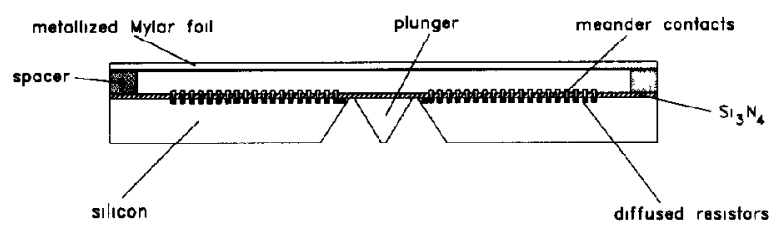

Fig 6 Cross-sectional view of the applanation sensor

certain area of the gold pattern as a function of the applied force, thus shortcircuiting the meander resistor arrays within that area Measuring the resulting array resistances thus reflects the shape of the flattened area, from which the diameter and the distance between the centre of the sensor and the nearest edge of the flattened area can easily be calculated With a prototype sensor a resolution of 60 and $105 \mu \mathrm{m}$ could be achieved for both parameters, respectively, which is more than enough for the tonometer application

By attaching two intracranıal pressure sensors to the applanation sensor in the configuration as shown in Fig 7, both measurng principles, according to Mackay-Marg as well as to Goldmann, can be performed One sensor is contacting the plunger of the applanation sensor with its boss, thus acting as a pressure sensor in the centre of the construction In this way a Mackay-Marg measurement can be realized, with simultaneous monitoring of the validity conditions The second sensor is contacting the total construction as 


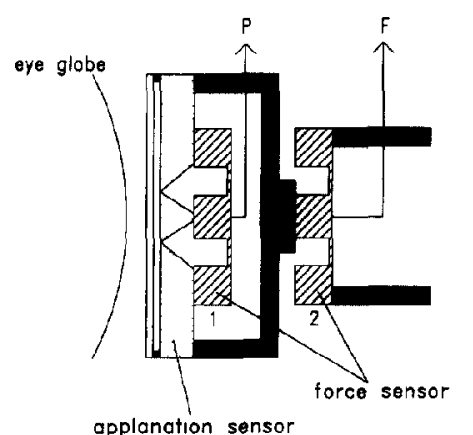

Fig 7 Sensor configuration of the Mackay-Marg as well as the Goldmann tonometer

described above with its boss, thus acting as a force sensor, necessary to use the system as a Goldmann tonometer The applanation sensor is in this case used to adjust the required diameter of $306 \mathrm{~mm}$

Experumental results of prototype systems, tested to measure the internal variable pressure of an artificial eye, have shown that both types of measurements can be performed within the required resolution It is believed that the possibility to measure simultaneously the $I O P$ with two principles is of great importance for ophthalmologists, due to the necessity of measuring the $I O P$ also in eyes with an aberrant cornea, in which case the measuring principle to be chosen is not a proor determined

Because the system provides the user with an electronic read-out of the applanation condition, it is much easier to use than the onginal optical procedure of the Goldmann tonometer Therefore the new system may also be useful for application by less specialized and experienced medical professionals

\section{Conclusions}

In the previous sections two types of biomedical sensors have been described, for which the application of silicon as a base material is essential These are only examples of what is possible, with arguments why silicon is used In the microphone concept a full integration of the sensor and the electronic circuitry appears to be necessary to improve the signal-to-noise ratio In the $I O P$ sensor a hybrid integration was chosen, based on the fact that cheap and reliable force sensors already exist and full integration should only complicate the technological processes involved Nevertheless the integration of one of the force sensors with the plunger in the applanation sensor, resulting in an integrated central pressure sensor, may be a useful future development
Hopefully the descnption may also have tnggered the reader to find the right solution to his particular measurnng problem in applying silicon and slicon technology

\section{Acknowledgements}

Makang proposals for $\mathrm{Ph} \mathrm{D}$ projects is only useful If one has the disposal of students who are able to pick up the idea and translate it into action In that sense the author gratefully acknowledges his former $\mathrm{Ph} D$ students Ad Sprenkels, Armand van der Donk, Patrick Scheeper and Kees den Besten for their continuous interest and perseverance in developing the described sensors The projects were financially supported by the Dutch Foundation for Fundamental Research on Matter (FOM) and the Dutch Technology Foundation (STW)

\section{References}

1 A G H van der Donk, I A Voorthuyzen and P Bergveld, General considerations of noise in microphone preamplifiers, Sensors and Actuators A, 25-27 (1991) 515-520

2 A G H van der Donk, $\mathbf{P}$ Bergveld and J A Voorthuyzen, Optımal desıgn of an electret microphone metal-oxide-semiconductor field-effect transistor preamplifier, $J$ Acoust SaC Am, 91 (1992) 2261-2269

3 A J Sprenkels, A silicon subminiature electret microphone, Ph $D$ Thests, University of Twente, Feb 18, 1988

4 A J Sprenkels, R A Groothengel, A J Verloop and P Bergveld, Development of an electret microphone in slicon, Sensors and Actuators, 17 (1989) 509-512

5 J A Voorthuyzen, K. Keskin and P Bergveld, Investıgations on the surface conductivity of silicon dioxide and methods to reduce it, Surf Sch, 187 (1987) 201-211

6 P R Scheeper, A silıcon condenser microphone materials and technology, $P h D$ Thesis, Unversity of Twente, Apr 8, 1993

7 P R Scheeper, J A Voorthuyzen, W Olthuis and P Bergveld, Investigation of attractive forces between PECVD silicon nitnde microstructures and an oxadized slicon substrate, Sensors and Actuators A, 30 (1992) 231-239

8 P R Scheeper, W Olthus and P Bergveld, A silicon condenser microphone with a silicon nitride diaphragm and backplate, $J$ Mucromech. Eur, 2 (1992) 187-189

9 PR Scheeper, AGH van der Donk, W Olthus and P Bergveld, Fabrication of silicon condenser microphones using single wafer technology, $J$ Microelectromech Systems, 1 (1992) $147-154$

10 A G H van der Donk, A silicon condenser microphone modelling and electronic circuitry, $P h . D$ Thesis, University of Twente, Aug 28, 1992

11 P R Scheeper, W Olthus and P Bergveld, Improvement of the performance of microphones with a silicon nitride diaphragm and backplate, Sensors and Actuators $A$, to be published 
12 P R Scheeper, P Bakker, W Olthuis and P Bergveld, The design, fabrication and testing of corrugated slicon nitnde diaphragms, J Microelectromech Systems, accepted for publication

$13 \mathrm{C}$ den Besten, Sensor systems for the measurement of intraocular pressure, $P h D$ Thesis, University of Twente, Apr 2, 1993
$14 \mathrm{C}$ den Besten and $\mathrm{P}$ Bergveld, A positioning sensor for tonometric applıcations, Sensors and Actuators A, 31 (1992) 24-28

$15 \mathrm{C}$ den Besten and $\mathrm{P}$ Bergveld, A new tonometer based on the application of micro-mechanical sensors, Proc IEEE Microelectromechanical Systems, Ft Lauderdale, FL, Feb 7-10, 1993, pp 105-110 